\title{
Variable cross-reactivity of Pseudomonas aeruginosa lipopolysaccharide- core-specific monoclonal antibodies and its possible relationship with serotype
}

\author{
Shin-ICHI Yokota, ${ }^{1}$ Masazumi Terashima, ${ }^{2}$ Joe ChiBa ${ }^{3}$ and Hiroshi Noguchi ${ }^{1 *}$ \\ ${ }^{1}$ Biotechnology Laboratory, Takarazuka Research Center, Sumitomo Chemical Co. Ltd, Takarazuka, Hyogo 665, Japan \\ ${ }^{2}$ Research Laboratories, Sumitomo Pharmaceuticals Co. Ltd, Osaka 554, Japan \\ ${ }^{3}$ Department of Biological Science and Technology, Faculty of Basic Technology, Science University of Tokyo, \\ Chiba 278, Japan
}

(Received 14 May 1991; revised 16 August 1991; accepted 7 October 1991)

\begin{abstract}
The core region of Pseudomonas aeruginosa lipopolysaccharide (LPS) was analysed by four LPS-core-specific human monoclonal antibodies (mAbs; FK-2E7, MH-4H7, OM-1D6 and NM-3G8). Reactivity of these mAbs to about $180 P$. aeruginosa strains was tested. FK-2E7 bound to strains of Homma serotype $E$ and $I$ at a frequency of about $90 \%$, to strains of serotype $M$ at about $50 \%$, and to strains of serotype $A$ and $G$ at about $30 \%$. MH-4H7 bound to $P$. aeruginosa strains of serotype $A, F, G, H, K$ and $M$ at a high frequency (45-87\%), but did not bind to any strains of serotype B, C, E and I. OM-1D6 and NM-3G8 bound to $P$. aeruginosa strains in a nearly serotypespecific manner. OM-1D6 reacted with all strains of serotype $G$ so far tested, and a few strains of serotype $M$. Furthermore, L-rhamnose in the LPS core of serotype $G$ was an immunodominant sugar recognized by OM-1D6 as an epitope. NM-3G8 bound to only a few strains of serotype $B$ and $M$. The variable reactivity of these mAbs suggests that antigenic heterogeneity of the LPS core is somewhat related with (O-polysaccharide-based) serotype. Among these mAbs, MH-4H7 and OM-1D6 showed a high level of protective activity against $P$. aeruginosa in an experimental infection model using normal mice. In vivo protective activity was shown to be closely related to in vitro binding activity to whole cells as determined by agglutination and flow cytometry, but not ELISA.
\end{abstract}

\section{Introduction}

Pseudomonas aeruginosa is a major pathogen of opportunistic infections. P. aeruginosa lipopolysaccharide (LPS), as well as that of the Enterobacteriaceae, consists of three regions, namely $\mathrm{O}$-polysaccharide, core oligosaccharide and lipid A (Wilkinson, 1983). These regions are distinguished by chemical constituents and antigenic properties. It is well known that $\mathrm{O}$-antigenic specificity, namely $\mathrm{O}$-serotype, is determined by the structure of the $\mathrm{O}$-polysaccharide and the endotoxic principle is expressed by lipid A (Luderitz et al., 1982).

In enterobacterial LPS, the core region is divided into two parts, the outer core and inner core. Generally, the inner core region contains characteristic sugars, 3-deoxyoctulosonic acid and heptose (Luderitz et al., 1982;

* Author for correspondence. Tel. 79774 2063; fax 797742133.

Abbreviations: LPS, lipopolysaccharide; mAb, monoclonal antibody.
Rietschel et al., 1985). The immunological properties, as well as the chemical constituents of the inner core region are expected to be common to all Gram-negative bacteria, including $P$. aeruginosa (Brade \& Galanos, 1983; Brade \& Brade, 1985). The outer core region exists between the O-polysaccharide and the inner core, and consists of about ten sugar residues. In $P$. aeruginosa, the outer core region commonly contains L-rhamnose, Dglucose, D-galactosamine and L-alanine, but the molar ratio of these constituents seems to be heterogeneous between strains (Wilkinson, 1983). However, the detailed chemical structure of this region has been reported only for strain NCTC1999 (Drewry et al., 1975), and PAC1 R and its defective mutants (Rowe \& Meadow, 1983), and their proposed partial structures were different from each other. Thus, the extent of the heterogeneity of the LPS core region is still obscure.

Our recent study (Yokota et al., 1989) demonstrated that human monoclonal antibodies (mAbs) $\mathrm{MH}-4 \mathrm{H} 7$ and $\mathrm{KN}-2 \mathrm{~B} 11$, recognizing the LPS outer core region, 
bound to $P$. aeruginosa strains of particular serotypes, namely A, F, G, H, K and M (classification of Homma, 1976), at a high frequency. Hence, we supposed that the heterogeneity of the outer core region of $P$. aeruginosa LPS might be related to the O-serotype. However, this result arose from only one epitope, recognized by MH-4H7 and $\mathrm{KN}-2 \mathrm{~B} 11$. In the present paper, we further characterized this heterogeneity by using four distinct types of LPS-core-specific human mAbs, including MH-4H7. Furthermore, biological activity of these mAbs was determined by in vitro and in vivo methods.

\section{Methods}

Bacterial strains. Homma serotype standard strains of $P$. aeruginosa (IID number) (Homma, 1976) were obtained from the Institute of Medical Science, University of Tokyo, Tokyo, Japan. The international antigenic typing scheme (Liu et al., 1983) and Fisher serotype (Fisher et al., 1969) standard strains (ATCC number) were obtained from the American Type Culture Collection, Rockville, Maryland, USA. $P$. aeruginosa PAC1, PAC1R (Habs serotype 3 ) and their LPSdefective mutant strains (Rowe \& Meadow, 1983) were a kind gift from P. M. Meadow, University College of London, London, UK. Clinical isolates of $P$. aeruginosa (SP number) were stocked in our laboratory, and serotyping was carried out by the slide agglutination method with a serotype grouping kit, Mei-assay (Meiji Seika Kaisha).

Preparation of LPS. P. aeruginosa was cultured in heart infusion broth (Nissui Pharmaceuticals) to late exponential-phase. LPS was extracted from bacterial cells by Triton X-100 according to the method of Uchida \& Mizushima (1987). The resulting LPS fraction was treated with $50 \mu \mathrm{g}$ proteinase $\mathrm{K} \mathrm{ml}^{-1}$ (Sigma) at $60^{\circ} \mathrm{C}$ for $1 \mathrm{~h}$, and then LPS was recovered by centrifugation $(100000 \mathrm{~g}, 90 \mathrm{~min})$ and ethanol precipitation. Protein and nucleic acid contents were less than $0.3 \%$ in these preparations.

Human mAbs. Human-mouse heterohybridoma cell lines producing human mAbs were established basically as previously described (Yokota et al., 1989). Human peripheral blood lymphocytes were prepared from heparinized blood of a healthy adult donor with a high antibody titre for $P$. aeruginosa immunogens. The lymphocytes were stimulated in vitro by culturing with $0.1 \%$ pokeweed mitogen (Gibco) and $0.0002 \%$ formalin-killed $P$. aeruginosa cells for $6 \mathrm{~d}$, and then fused with a human-mouse heteromyeloma using polyethylene glycol. Specific antibody production was screened by ELISA using plates coated with whole $P$. aeruginosa cells of some serotype standard strains (A, B, E, G, I and M).

Four LPS-core-specific human mAbs, MH-4H7, FK-2E7, OM-1D6 and NM-3G8, and one lipid A-specific human mAb, NM-3D7, were obtained. FK-2E7 was $\operatorname{IgM} \kappa$, and the other human mAbs were $\operatorname{IgM} \lambda$. The parental heteromyeloma cell line was a triple hybridoma (3HL36J-C5) for establishing hybridoma OM-1D6, and a heterohybridoma (SHM-D33) (Teng et al., 1983) was used to establish the other mAbproducing hybridoma cell lines.

The hybridoma was adapted to a serum-free medium, Celgrosser $\mathbf{H}$ (Sumitomo Pharmaceuticals). IgM was purified by gel chromatography on a column of Superose 6 (Pharmacia LKB) in Dulbecco's PBS from a serum-free culture supernatant.

ELISA. The methods for ELISA and preparation of bacterial-cellcoated plates were described previously (Yokota et al., 1989). LPScoated plates were prepared according to the method of Pollack et al. (1987). Alkaline-phosphatase-conjugated goat anti-human IgM anti- body (Kirkegaad \& Perry Laboratories Inc.) and sodium p-nitrophenylphosphate were used as secondary antibody and substrate, respectively. Binding activity was expressed as $A_{405}$.

For competitive ELISA, the antibody solution and competitor solution were mixed, preincubated for $37^{\circ} \mathrm{C}$ for $1 \mathrm{~h}$, and then subjected to ELISA.

$S D S-P A G E$ and Western blotting. Each LPS preparation $(5 \mu \mathrm{g})$, all of which were treated with proteinase $K$, was subjected to SDS-PAGE using a $10-20 \%(w / v)$ polyacrylamide gradient gel (Daiichi Chemicals) according to the method of Laemmli (1970). LPS was visualized by silver staining according to the method of Tsai \& Frasch (1982). Western blotting was carried out as described previously (Yokota et al., 1989). Briefly, LPS on the gels was transferred to a PVDF filter (Millipore). The filter was blocked with $2 \%(\mathrm{w} / \mathrm{v})$ casein in PBS, and subsequently incubated with $\mathrm{mAb}\left(1 \mu \mathrm{g} \mathrm{ml}^{-1}\right)$ and alkaline-phosphatase conjugated goat anti-human IgM antibody (1000-fold dilution). Specific binding was detected with sodium 5-bromo-4-chloro-3indoylphosphate $\left(0.15 \mathrm{mg} \mathrm{ml}^{-1}\right)$ and nitroblue tetrazolium chloride $\left(0.3 \mathrm{mg} \mathrm{ml}^{-1}\right)$ in $100 \mathrm{~mm}$-sodium carbonate buffer ( $\mathrm{pH} \mathrm{9.8)} \mathrm{containing}$ $1 \mathrm{mM}-\mathrm{MgCl}_{2}$.

Flow cytometry. Flow cytometry was carried out basically according to the method of Evans et al. (1990). P. aeruginosa cells were cultured in heart infusion broth to late exponential-phase, washed with PBS and fixed with $1 \%(\mathrm{v} / \mathrm{v})$ formaldehyde at $25^{\circ} \mathrm{C}$ for $1 \mathrm{~h}$. Washed cells were resuspended in PBS to an $A_{600}$ of 0.5 (approximately $1 \times 10^{8}$ c.f.u. $\left.\mathrm{ml}^{-1}\right)$. The suspension $(0.5 \mathrm{ml})$ was mixed with a $5 \mu \mathrm{g}$ $\mathrm{mAb} \mathrm{ml} l^{-1}$ solution $(0.5 \mathrm{ml})$, and then incubated at $37^{\circ} \mathrm{C}$ for $1 \mathrm{~h}$. The resulting samples were washed twice with PBS, suspended in $0.5 \mathrm{ml}$ fluorescein isothiocyanate (FITC)-conjugated goat anti-human IgM antibodies (Tago) 100 -fold diluted with PBS, and incubated at $37^{\circ} \mathrm{C}$ for $1 \mathrm{~h}$. Each sample was prepared in triplicate. Flow cytometry was performed with FACScan (Becton-Dickinson), equipped with a $15 \mathrm{~mW}$ argon ion laser. Stained bacterial cells were suspended in PBS at a concentration of about $10^{6} \mathrm{c.f}$.u. $\mathrm{ml}^{-1}$, and 20000 stained cells were totally evaluated in each analysis. Bacterial aggregates and background noise were excluded with the use of gates on the light scatter signals.

Protective activity against $P$. aeruginosa infection. The experimental infection model using normal mice was as described previously (Terashima et al., 1991). Four-week old male ICR mice (Japan SLC Inc.) (22-24 $\mathrm{g}$ body wt.) were used. Briefly, a cell suspension $(0.2 \mathrm{ml})$ of each bacterial strain containing $5 \%(\mathrm{w} / \mathrm{v})$ mucin (Difco) was injected intraperitoneally into ten mice per group. One hour later, $0.2 \mathrm{ml} \mathrm{mAb}$ in PBS was administered intraperitoneally at a dosage of 0.1 or $1.0 \mu \mathrm{g}$ per mouse. As a control group, BSA (10 $\mu \mathrm{g}$ per mouse) was administered instead of $\mathrm{mAb}$. The mice were observed for $6 \mathrm{~d}$. The lethal dosage of bacteria causing death in $50 \%$ mice $\left(\mathrm{LD}_{50}\right)$ was calculated by probit analysis. The significance of difference from the control group was indicated by non-overlapping $95 \%$ confidence intervals.

Other materials and methods. Agglutination activity using viable bacterial cells was determined as described previously (Terashima $e t$ $a l ., 1991)$. Other materials and methods were the same as those described in previous papers (Yokota et al., 1989, 1990).

\section{Results}

Binding to various serotypes of P. aeruginosa in ELISA

The binding profile of mAbs was determined by ELISA using whole bacterial cells as a solid antigen (Table 1). MH-4H7 $(\mu, \lambda)$ and FK-2E7 $(\mu, \kappa)$ cross-reacted to strains 
Table 1. Binding profile of anti-core $m A$ Abs to $P$. aeruginosa standard strains and clinical isolates

Whole cells of each strain were fixed to ELISA plates with glutaraldehyde. An $A_{405}$ value of $>0.15$ in ELISA (mean value from triplicate experiments) at a concentration of $1 \mu \mathrm{g}$ antibody $\mathrm{ml}^{-1}$ was considered to be binding-positive.

\begin{tabular}{|c|c|c|c|c|c|}
\hline \multirow{2}{*}{$\begin{array}{l}\text { Homma } \\
\text { serotype }\end{array}$} & \multirow{2}{*}{$\begin{array}{l}\text { No. of } \\
\text { strains } \\
\text { tested }\end{array}$} & \multicolumn{4}{|c|}{$\begin{array}{l}\text { No. of strains giving } \\
\text { positive binding }\end{array}$} \\
\hline & & FK-2E7 & MH-4H7 & OM-1D6 & NM-3G8 \\
\hline $\mathbf{A}$ & 21 & 7 & 15 & 0 & 0 \\
\hline B & 28 & 0 & 0 & 0 & 6 \\
\hline $\mathbf{C}$ & 8 & 0 & 0 & 0 & 0 \\
\hline $\mathrm{E}$ & 24 & 22 & 0 & 0 & 0 \\
\hline$F$ & 7 & 1 & 6 & 0 & 0 \\
\hline $\mathbf{G}$ & 23 & 8 & 20 & 23 & 0 \\
\hline $\mathbf{H}$ & 16 & 0 & 10 & 0 & 0 \\
\hline I & 23 & 21 & 0 & 0 & 0 \\
\hline $\mathrm{K}$ & 4 & 0 & 3 & 0 & 0 \\
\hline $\mathbf{M}$ & 22 & 10 & 10 & 4 & 2 \\
\hline NT & 6 & 2 & 1 & 1 & 1 \\
\hline
\end{tabular}

belonging to some serotypes. FK-2E7 bound to strains of serotype $\mathrm{E}$ and $\mathrm{I}$ at a frequency of about $90 \%$, serotype $\mathrm{M}$ at about $50 \%$, serotype $A$ and $G$ at about $30 \%$. MH-4H7 bound to strains of serotype $A, F, G, H$ and $K$ with a high frequency $(63-87 \%)$, to serotype $M$ at about $50 \%$, but did not react with any strains of other serotypes. OM-1D6 $(\mu$, d) bound to $100 \%$ of serotype $G$ strains tested and a few strains of serotype $\mathbf{M}$, but did not bind to strains of any other serotypes. NM-3G8 $(\mu, \lambda)$ bound to only a few strains of serotype $B$ and $M$.

\section{Western blotting analysis}

LPS preparations were subjected to SDS-PAGE and Western blotting. All four mAbs specifically bound to a relatively low-molecular-mass population of each LPS preparation which was isolated from a binding-positive strain in ELISA (Fig. 1). Namely, MH-4H7 stained lowmolecular-mass LPSs derived from serotype $A$ and $G$, but not serotype B (Fig. 1c). FK-2E7 specifically stained LPSs derived from IID1130 (serotype E) and IID1010 (serotype I) (data not shown), as well as LPS derived from serotype A and G as shown in Fig. 1(b). OM-1D6 specifically stained serotype G LPS (Fig. $1 d$ ). NM-3G8 only stained LPS derived from IID1002 (serotype B), and gave a much weaker reaction in the immunoblot assay than the other mAbs (Fig. 1e).

\section{Binding to LPS-defective mutants}

Binding activity of anti-core mAbs to LPS-defective mutants derived from $P$. aeruginosa PAC1 and PAC1R (Rowe \& Meadow, 1983), of which LPS core structures had been well defined, was determined by ELISA using whole cells as a solid antigen (Table 2). First, we determined the serotype of these strains according to Homma's classification by agglutination testing using mAbs (Mei-assay). Whereas the smooth type strains were classified into serotype A, serologically related to Habs serotype 3, the semirough and rough strains derived from PAC1R were classified into serotype $M$. Human anti-lipid A mAb NM-3D7 was used as a control in this study. NM-3D7 specifically bound to the lipid A portion of LPS derived from Salmonella and Escherichia coli, as well as $P$. aeruginosa (data not shown). The mAb NM-3D7 bound to rough strains more strongly than to smooth strains. FK-2E7 reacted more strongly with rough-natured strains, PAC608 and PAC557, but reacted weakly with deep rough strains PAC556 and PAC611. In contrast, $\mathrm{MH}-4 \mathrm{H} 7$ did not react at all to these deep rough strains PAC556 and PAC611, as previously reported (Yokota et al., 1989). PAC556 is reported to lack only the L-rhamnose residue of the LPS outer core region compared with PAC557 (Rowe \& Meadow, 1983). The other anti-core mAbs, OM-1D6 and NM-3G8, were not observed to bind to a series of strains, including a parental strain of serotype $\mathrm{A}$, at all.

\section{Competition of monosaccharide with $m A b$ binding}

Competition experiments with monosaccharide were performed by ELISA using LPS-coated plates. All mAbs bound to SP10067 (serotype M) and IID1020 (serotype G), except that NM-3G8 did not react to IID 1020 . L-Rhamnose and methyl-L-rhamnoside (the preparation contained $90 \% \alpha$-anomer) inhibited the binding of both 


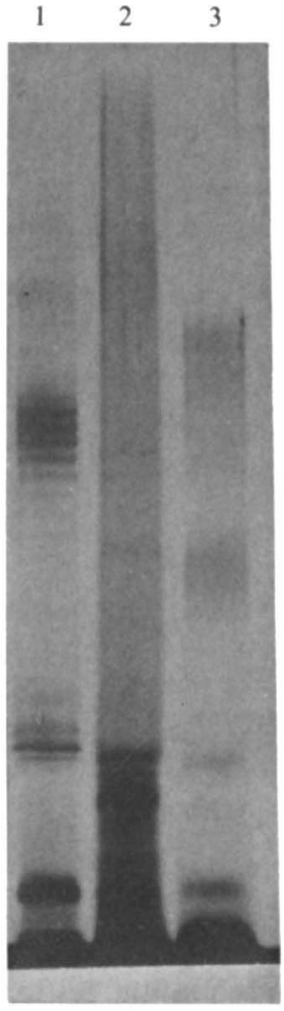

(a)

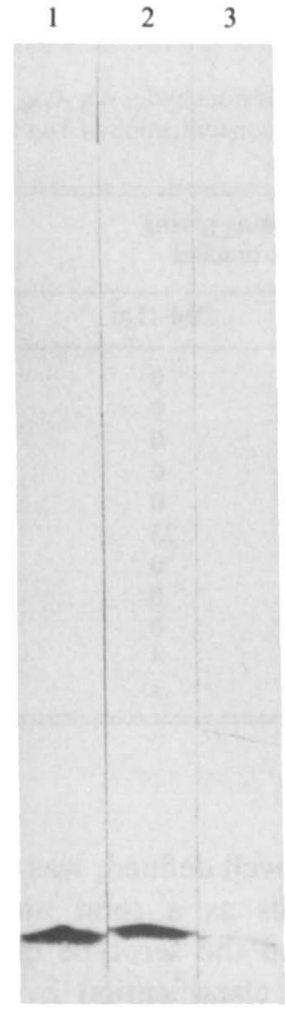

(b)

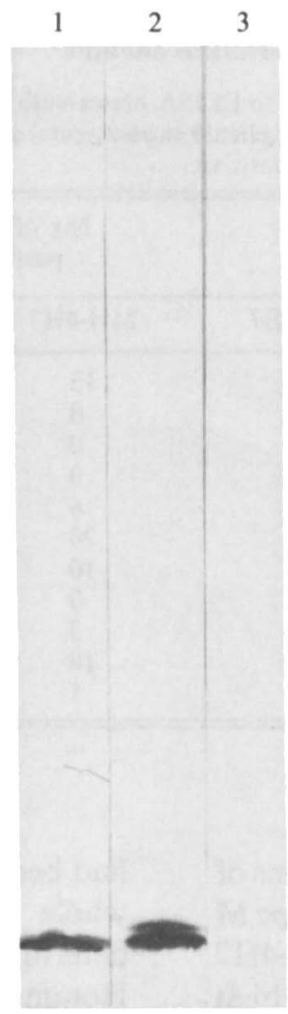

(c)

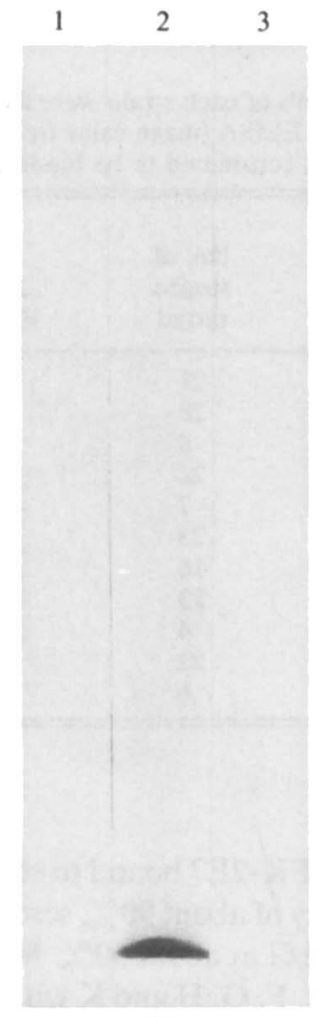

$(d)$

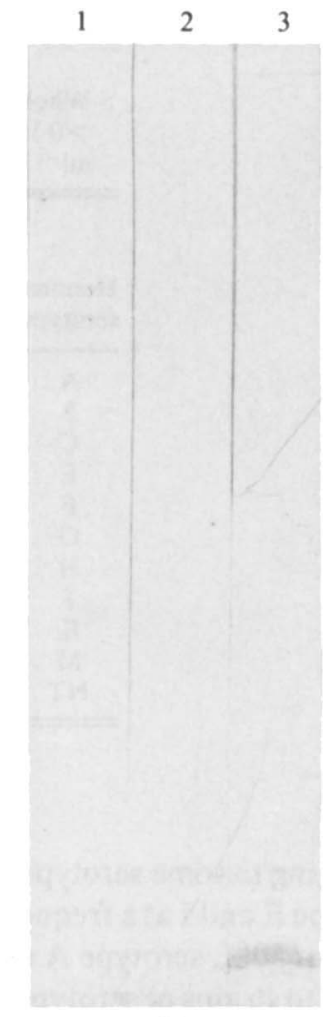

(e)

Fig. 1. SDS-PAGE and Western blotting analysis of $P$. aeruginosa LPS preparations with human mAbs. The LPS preparations, which were treated with proteinase K, were derived from $P$. aeruginosa IID1001 (serotype A; lane 1), IID1020 (serotype G; lane 2) and IID1002 (serotype B; lane 3). To each lane, $5 \mu \mathrm{g}$ sample was applied. Silver staining pattern of each LPS preparation $(a)$ and immunoblots with (b) FK-2E7, (c) MH-4H7, (d) OM-1D6 and (e) NM-3G8 are shown.

Table 2. Binding of anti-LPS-core $m A$ Abs and anti-lipid $A$ mAb to P. aeruginosa $P A C 1 R$ and its LPS-defective mutant strains

Binding activity was determined by ELISA using plates coated with whole cells of each strain and expressed as mean $A_{405}$ values from triplicate experiments.

\begin{tabular}{lccccccc}
\hline \hline & & \multicolumn{5}{c}{ Binding activity $\left(A_{405}\right)$} \\
\cline { 5 - 8 } Strain & Chemotype* & Serotype & FK-2E7 & MH-4H7 & OM-1D6 & NM-3G8 & NM-3D7 \\
\hline PAC1R & S & A & $0 \cdot 15$ & 0.25 & 0 & 0 & 0.02 \\
PAC609 & S & A & 0.18 & 0.28 & 0 & 0 & 0.05 \\
PAC608 & SR & M & 1.48 & 1.11 & 0 & 0 & 0.13 \\
PAC557 & R & M & 1.62 & 1.13 & 0 & 0 & 0.49 \\
PAC556 & R & M & 0.38 & 0.02 & 0 & 0 & 0.74 \\
PAC611 & R & M & 0.20 & 0.04 & 0 & 0 & 0.85 \\
\hline \hline
\end{tabular}

*S, smooth; SR, semirough; R, rough.

OM-1D6 and $\mathrm{MH}-4 \mathrm{H} 7$ to LPS derived from both IID1020 (serotype G) and SP10067 (serotype M). Other monosaccharides, such as L-fucose, D-fucose, D-quinovose, D-mannose, D-galactose, D-glucose, D-glucuronic acid, $N$-acetyl-D-glucosamine, $N$-acetyl-D-galactosamine and $\mathrm{N}$-acetylneuraminic acid, did not inhibit the binding of OM-1D6 and $\mathrm{MH}-4 \mathrm{H} 7$ even at concentrations of $500 \mathrm{mM}$. A much lower concentration of $\mathrm{L}$-rhamnose and methyl-L-rhamnoside inhibited the binding of OM-1D6 compared with $\mathrm{MH}-4 \mathrm{H} 7$ (Fig. 2). Inhibition of the 
Table 3. Comparison in binding activity of anti-LPS core mAbs to whole bacterial cells determined by ELISA, agglutination and flow cytometry

\begin{tabular}{|c|c|c|c|c|c|}
\hline $\mathrm{mAb}$ & Strain & Serotype & $\begin{array}{l}\text { ELISA } \\
\left(A_{405}\right)\end{array}$ & $\begin{array}{l}\text { Agglutinating } \\
\text { activity } \\
\left.(\mu \mathrm{g} \mathrm{ml})^{-1}\right)^{*}\end{array}$ & $\begin{array}{l}\text { Flow cytometry } \\
\text { (percentage positive } \\
\text { fluorescence } \dagger \text { ) }\end{array}$ \\
\hline FK-2E7 & $\begin{array}{l}\text { SP6783 } \\
\text { SP9759 } \\
\text { SP10046 } \\
\text { SP6788 } \\
\text { SP10067 } \\
\text { SP6764 } \\
\text { IID5018 }\end{array}$ & $\begin{array}{l}\text { A } \\
\text { E } \\
\text { I } \\
\text { G } \\
\text { M } \\
\text { M } \\
\text { M }\end{array}$ & $\begin{array}{l}0 \\
1 \cdot 72 \\
1.68 \\
1 \cdot 76 \\
1.98 \\
2 \cdot 10 \\
0\end{array}$ & $\begin{array}{l}>25 \\
>25 \\
>25 \\
>25 \\
>25 \\
>25 \\
>25\end{array}$ & $\begin{array}{r}2 \cdot 0 \\
10 \cdot 2 \\
22 \cdot 3 \\
18 \cdot 2 \\
60 \cdot 0 \\
26 \cdot 0 \\
3 \cdot 1\end{array}$ \\
\hline $\mathrm{MH}-4 \mathrm{H} 7$ & $\begin{array}{l}\text { SP6783 } \\
\text { SP6788 } \\
\text { SP9792 } \\
\text { SP10067 } \\
\text { SP6764 } \\
\text { IID5018 }\end{array}$ & $\begin{array}{l}\mathrm{A} \\
\mathrm{G} \\
\mathrm{G} \\
\mathbf{M} \\
\mathbf{M} \\
\mathbf{M}\end{array}$ & $\begin{array}{l}1 \cdot 10 \\
2 \cdot 44 \\
1.98 \\
2 \cdot 19 \\
2 \cdot 24 \\
0\end{array}$ & $\begin{array}{r}0.47 \\
0.94 \\
0.94 \\
0 \cdot 12 \\
15 \\
25\end{array}$ & $\begin{array}{r}64.9 \\
74.9 \\
74.9 \\
71.9 \\
59 \cdot 3 \\
1.5\end{array}$ \\
\hline OM-1D6 & $\begin{array}{l}\text { SP6783 } \\
\text { SP6788 } \\
\text { SP9792 } \\
\text { SP10067 } \\
\text { SP6764 } \\
\text { IID5018 }\end{array}$ & $\begin{array}{l}A \\
G \\
G \\
M \\
M \\
M\end{array}$ & $\begin{array}{l}0 \\
2 \cdot 20 \\
1 \cdot 85 \\
2 \cdot 10 \\
0 \cdot 23 \\
0\end{array}$ & $\begin{array}{l}>25 \\
0.47 \\
0 \cdot 94 \\
0 \cdot 12 \\
>25 \\
>25\end{array}$ & $\begin{array}{r}0.9 \\
97 \cdot 6 \\
95 \cdot 2 \\
92.9 \\
5.9 \\
1.5\end{array}$ \\
\hline NM-3G8 & $\begin{array}{l}\text { SP10067 } \\
\text { IID1002 }\end{array}$ & $\begin{array}{l}\text { M } \\
\text { B }\end{array}$ & $\begin{array}{l}0.13 \\
0.26\end{array}$ & $\begin{array}{l}>25 \\
>25\end{array}$ & $\begin{array}{r}31.4 \\
9 \cdot 6\end{array}$ \\
\hline
\end{tabular}

* The value represents the minimum concentration of $\mathrm{mAb}$ required for visible agglutination.

+ Value represents the percentage of bacterial cells giving a positive fluorescence intensity over the background level at the level of sensitivity shown in Fig. 2.

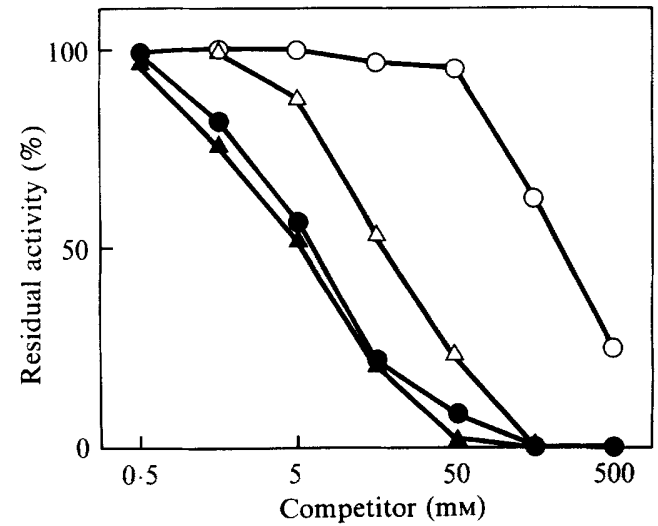

Fig. 2. Competition of L-rhamnose and methyl-L-rhamnoside with the binding of mAbs to $P$. aeruginosa SP10067 LPS in ELISA. OM-1D6 (O and $\Delta$ ) or $\mathrm{MH}-4 \mathrm{H} 7(\mathrm{O}$ and $\triangle)\left(1 \mu \mathrm{g} \mathrm{ml}^{-1}\right.$ each) were incubated with various concentrations of L-rhamnose $(O$ and $O$ ) or methyl-Lrhamnoside (containing $90 \% \alpha$-anomer; $\Delta$ and $\triangle$ ), and then applied to ELISA using the plate coated with LPS derived from SP10067 (serotype M). The results are expressed as a percentage of the $A_{405}$ reading compared with that without competitor. Mean values from triplicate experiments are shown. The $100 \% A_{405}$ values were 1.02 (MH-4H7) and 0.82 (OM-1D6).

binding of $\mathrm{MH}-4 \mathrm{H} 7$ by L-rhamnose was weaker than by methyl-L-rhamnoside. In contrast, FK-2E7 and NM$3 \mathrm{G} 8$ were not observed to compete in binding to LPS derived from IID1020 (in the case of FK-2E7) and
SP10067 with any monosaccharides mentioned above, even at high concentrations of $500 \mathrm{mM}$.

\section{Cross reaction to other Gram-negative bacterial LPSs}

Cross-reaction of the anti-core mAbs to LPSs derived from various Gram-negative bacteria other than $P$. aeruginosa was tested by ELISA using LPS as a solid antigen. No cross reaction to any other bacterial LPSs tested was observed, except the reaction of $\mathrm{MH}-4 \mathrm{H} 7$ to E. coli $\mathrm{O} 26$ : B6 LPS as described previously (Yokota $e t$ al., 1990). Furthermore, anti-core mAbs were not observed to bind in ELISA to lipid A derived from E. coli $\mathrm{J} 5$, Salmonella minnesota $\mathrm{R} 595$ and $P$. aeruginosa IID1020.

Binding activity of $m A b$ to bacterial cells in flow cytometry and agglutination activity

We compared the binding activity in ELISA of each $\mathrm{mAb}$ to whole bacterial cells with the binding activity in flow cytometry and agglutination activity (Table 3 and Fig. 3). High agglutination activity was observed for MH-4H7 and OM-1D6, but not for FK-2E7 and NM$3 \mathrm{G} 8$ (Table 3). In flow cytometry analysis, $\mathrm{MH}-4 \mathrm{H} 7$ and OM-1D6 gave relatively high intensities of fluorescence 


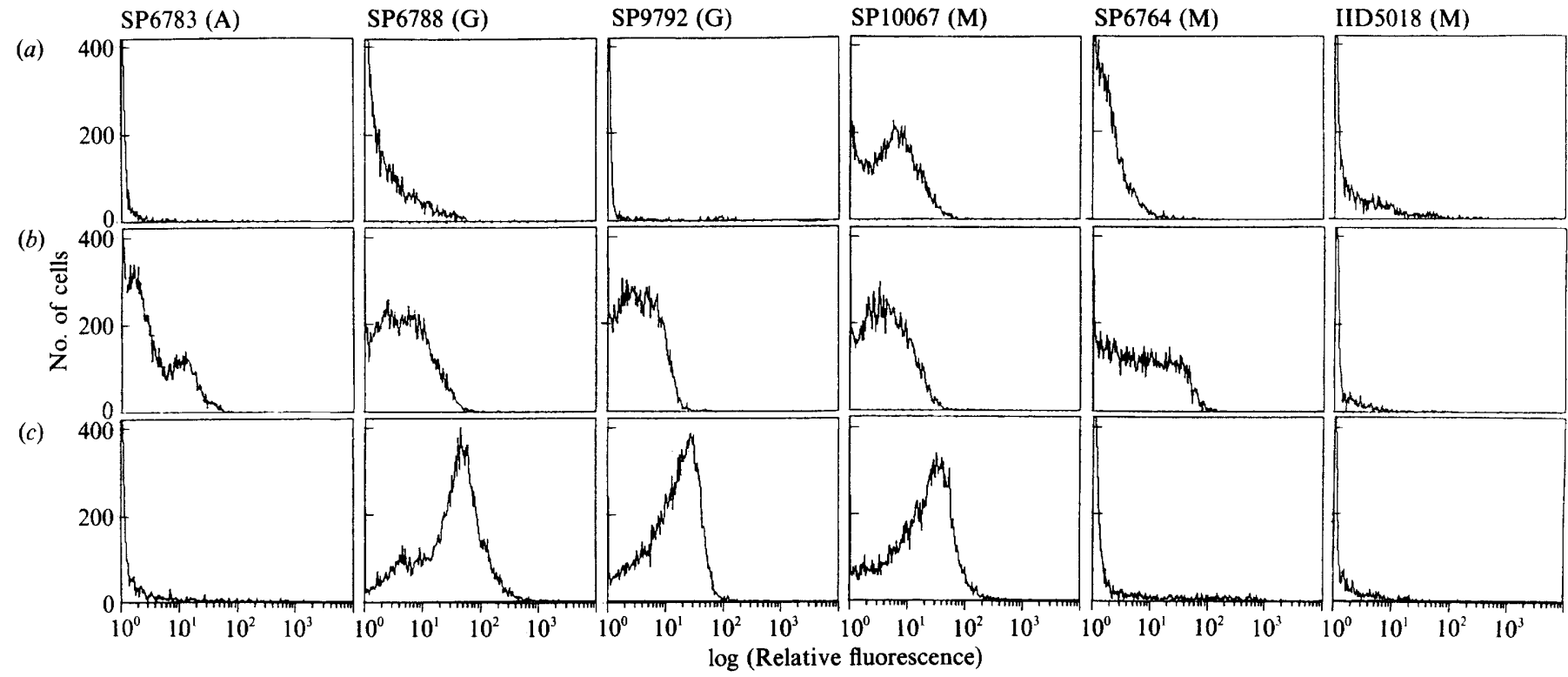

Fig. 3. Flow cytometry analysis of the binding of human anti-LPS-core mAbs, FK-2E7 (a), MH-4H7 (b) and OM-1D6 (c), to $P$. aeruginosa. Green fluorescence intensity of the mAbs with whole cells of $P$. aeruginosa is expressed as a log scale on the horizontal axis. The number of cells giving an intensity of green fluorescence is indicated on the vertical axis. The name of each strain is shown at the top of the traces and the serotype of each strain used is shown in parentheses.

Table 4. Protective activity of human $m A$ Abs against $P$. aeruginosa infection in experimental mouse model

\begin{tabular}{ccccccc}
\hline \hline mAb & $\begin{array}{c}\text { Dose } \\
\text { administered } \\
(\mu \mathrm{g} \text { per mouse) }\end{array}$ & $\begin{array}{c}\text { Strain } \\
\text { challenged }\end{array}$ & Serotype & Control & mAb-treated & $\begin{array}{c}\text { LD } \\
\text { Protection* } \\
\text { (-fold) }\end{array}$ \\
\hline MH-4H7 & $0 \cdot 1$ & SP6788 & G & $3.0 \times 10^{4}$ & $3.2 \times 10^{5}$ & $11 \dagger$ \\
& $0 \cdot 1$ & SP9792 & G & $5.0 \times 10^{5}$ & $1.6 \times 10^{7}$ & $32 \dagger$ \\
& $1 \cdot 0$ & SP6783 & A & $4.0 \times 10^{5}$ & $3.2 \times 10^{6}$ & $8 \cdot 0 \dagger$ \\
OM-1D6 & $1 \cdot 0$ & SP7514 & H & $1.5 \times 10^{6}$ & $6.6 \times 10^{6}$ & $4.4 \dagger$ \\
& $0 \cdot 1$ & SP6788 & G & $3.6 \times 10^{4}$ & $>3.4 \times 10^{5}$ & $>9.4 \dagger$ \\
FK-2E7 & $0 \cdot 1$ & SP9792 & G & $2.6 \times 10^{5}$ & $6.3 \times 10^{6}$ & $24 \dagger$ \\
& $1 \cdot 0$ & SP9759 & E & $1.2 \times 10^{5}$ & $1.4 \times 10^{5}$ & 1.2 \\
& $1 \cdot 0$ & SP10046 & I & $1.2 \times 10^{5}$ & $1.1 \times 10^{5}$ & 0.9 \\
\hline \hline
\end{tabular}

* $\mathrm{LD}_{50}$ (mAb-treated)/LD $\mathrm{LD}_{50}$ (control).

† Significantly different $(P<0.05)$ from control group.

against both smooth and rough (serotype $\mathbf{M}$ ) strains in parallel with the binding activity observed in ELISA (Fig. 3b,c). OM-1D6 gave a relatively higher intensity of fluorescence than MH-4H7. On the other hand, FK-2E7 did not exhibit a high intensity fluorescence with strains to which it bound at high frequency in ELISA, except with SP10067 (serotype M) [Fig. 3 (a) and Table 3]. In the case of $\mathrm{MH}-4 \mathrm{H} 7$ and $\mathrm{FK}-2 \mathrm{E} 7$, apparently non-staining populations were observed when the level of sensitivity was as used in the experiments of Fig. 3 and Table 3. However, we observed that almost all populations showed significant fluorescence for ELISA-positive strains measured in conditions of higher sensitivity but not for ELISA-negative strains (data not shown).
NM-3G8 gave a very low intensity of fluorescence against serotype B strains, whereas it gave a relatively high intensity of fluorescence against serotype $\mathbf{M}$ strains, as did FK-2E7 (Table 3).

\section{Protective activity against experimental infection in mice}

In vivo protective activity of the human $\mathrm{mAb}(\operatorname{IgM})$ was assessed in an experimental infection model using normal mice (Table 4). MH-4H7 and OM-1D6 showed highly protective activity against infections with $P$. aeruginosa strains, which the mAbs bound to and caused agglutination in vitro. In contrast, FK-2E7 did not show any significant in vivo protective activity against 
strains to which the mAb bound in vitro. As mentioned before, FK-2E7 did not cause agglutination of the strains used.

\section{Discussion}

Four different types of human mAbs recognizing the $P$. aeruginosa LPS core region were prepared. The sites recognized by these mAbs were deduced to be in the core region from the following. (i) In Western blot analysis (Fig. 1), all the mAb preferentially reacted with a lowmolecular-mass population of LPS preparations. (ii) The mAbs did not bind to the lipid A preparation in ELISA (data not shown). (iii) In the case of FK-2E7 and MH$4 \mathrm{H} 7$, the mAbs bound strongly to rough mutants PAC608 and PAC557 at higher rate than a parental strain PAClR (Table 2). The mAbs bound weakly to PAC556 and PAC611, the core region of which was defective in part. In addition, cross reactivity to Gramnegative bacterial LPS was not observed with the exception of the cross-reaction of $\mathrm{MH}-4 \mathrm{H} 7$ to $E$. coli O26:B6 LPS, as reported previously (Yokota et al., 1990).

The core region, especially the outer core region, had been thought to be characteristic of, and common to $P$. aeruginosa. D-Galactosamine, L-rhamnose, D-glucose and $\mathrm{L}$-alanine are reported to be common constituents of the outer core (Wilkinson, 1983). However, a few researchers have pointed out that antibodies directed to the LPS core cross-react with different serotype strains of P. aeruginosa (Hancock et al., 1983; Fomsgaard et al., 1988; Yokota et al., 1989), and the cross-reactivity is likely to be caused by the heterogeneity of the LPS core region. A systematic study on LPS core antigens of $\boldsymbol{P}$. aeruginosa has not been carried out, because a detailed structural study on the LPS core of each strain is thought to be very laborious. In this study, we tried to analyse the LPS core region of $P$. aeruginosa by using four human mAbs and about $180 P$. aeruginosa strains including serotype standard strains and clinical isolates.

The mAbs FK-2E7 and MH-4H7 cross-reacted with strains belonging to some serotypes (Table 1). The epitope is likely to exist in some, but not all, of $P$. aeruginosa strains, and it might be distributed in a serotype-dependent manner. The epitope recognized by MH-4H7 was shared by a almost all strains belonging to serotype A, F, G, H, K and $M$. We have demonstrated that the L-rhamnose residue in the core region acts as an epitope to some extent, this being deduced from the results of a binding experiment with a series of LPSdefective mutants of PAC1R (Table 2) and a competition experiment with monosaccharide (Fig. 2). On the other hand, the epitope recognized by FK-2E7 was found on about $90 \%$ of serotype $\mathrm{E}$ and I strains, about $30 \%$ of serotype $A$ and $G$ strains, and about $50 \%$ of serotype $M$ strains. The binding profile of FK-2E7 to $P$. aeruginosa strains was different from $\mathrm{MH}-4 \mathrm{H} 7$, and L-rhamnose, as well as methyl-L-rhamnoside, did not compete with the binding of FK-2E7, in contrast to $\mathrm{MH}-4 \mathrm{H} 7$ even though the binding experiments using a series of LPS-defective mutants (Table 2) suggested that the epitope for FK-2E7 is located in the neighbourhood of that for $\mathrm{MH}-4 \mathrm{H} 7$, at least on P. aeruginosa PAC1R LPS. Two mAbs, OM-1D6 and NM-3G8, bound to $P$. aeruginosa strains in a nearly serotype-specific manner (Table 1). Thus, a characteristic epitope recognized by OM-1D6 was shared with $100 \%$ of serotype $\mathrm{G}$ strains tested, and OM-1D6 was shown to strongly react to the whole cells from the results of flow cytometry analysis (Fig. 3) and in vivo protective activity (Table 4). Furthermore, a competition experiment with monosaccharide (Fig. 2) suggests that Lrhamnose might be greatly involved as an epitope for OM-1D6. In other words, L-rhamnose seemed to be an immunodominant sugar in the LPS core region of serotype $G$ strains. Some serotype B and $M$ strains had an epitope recognized by NM-3G8, but other serotype strains did not. A line of results suggest that the heterogeneity of the LPS core of $P$. aeruginosa is related to (O-polysaccharide-based) serotype.

Both OM-1D6 and NM-3G8, which reacted in a nearly serotype-specific manner, with serotype $G$ and $B$, respectively, also reacted with some strains of serotype M. Serotype $\mathbf{M}$ strains were rough-natured, due to defective or decreased amounts of O-polysaccharides (unpublished results). Homma (1976) also reported that the standard strain of serotype $M$ was rough. Antigenic target(s) of serotype $M$ have not been clarified yet. In this study, we also observed that rough and semirough strains were classified as serotype $\mathbf{M}$ according to the serotype grouping kit (Table 2). Thus, serotype $M$ does not seem to be based upon the nature of the O-polysaccharide antigen. The results indicated that binding specificity of OM-1D6 and NM-3G8 was able to be regarded as serotype-specific.

Human mAbs directed against some common epitopes in the LPS core region are one of the candidates for antibody therapy against $P$. aeruginosa infections and will be used as an alternative to, or in combination with anti-O-polysaccharide mAbs. Two mAbs, $\mathrm{MH}-4 \mathrm{H} 7$ and OM-1D6, showed a high level of protective activity against $P$. aeruginosa infections in a normal mouse infection model (Table 4). We have recently reported details of in vivo protective activity of $\mathrm{MH}-4 \mathrm{H} 7$ (Terashima et al., 1991). Among these LPS-core-specific human mAbs, we compared in vivo protective activity with in vitro binding activity determined by different methods. Flow cytometry has recently been used for 
analysing bacterial cell surface antigens (Klebba et al., 1990; Evans et al., 1990; Nelson et al., 1990). Applying flow cytometry to our system (Fig. 2), OM-1D6 and $\mathrm{MH}-4 \mathrm{H} 7$, which showed a potent protective activity, were shown to give relatively higher fluorescence than FK-2E7 and NM-3G8. In the case of FK-2E7 and $\mathrm{MH}-4 \mathrm{H} 7$, the non-staining subpopulations were observed with a level of sensitivity as shown in Fig. 3 and Table 3. This observation is not likely to be due to the existence of a true non-staining subpopulation within an ELISApositive strain, because in measurement with a higher level of sensitivity, almost all bacterial polulations showed a significant degree of fluorescence by staining with the mAbs MH-4H7 and FK-2E7. However, ELISA-negative strains did not show any significant fluorescence. The in vivo protective activity was shown to correlate well with in vitro binding activity as determined by flow cytometry (Tables 3 and 4), and agglutination, but not by binding in ELISA. In ELISA, a significant difference of binding activity among mAbs was not observed in antibody dilution curve experiments (data not shown). Nelson et al. (1990) pointed out that ELISA has tended to over-emphasize the binding of anti-core $\mathrm{mAbs}$ to whole bacterial cells in contrast to flow cytometry. In our study, a similar phenomenon was observed. The physical state of bacteria in a cell suspension was probably different from that on a solid surface. Thus, ELISA does not seem to be a suitable method for evaluating true binding activity, which would predict in vivo activity, among different antibodies.

A line of evidence suggested that the epitopes in the LPS core recognized by MH-4H7 and OM-1D6 were likely to be exposed on the cell surface to a greater extent than those for FK-2E7 and NM-3G8. In conclusion, the heterogeneity of the LPS core region of $P$. aeruginosa might be related to (O-polysaccharide-based) serotype, and some epitopes in the LPS core would be valuable and useful as protective antigens.

The authors thank Dr Pauline M. Meadow for providing $P$. aeruginosa mutant strains, Kenji Irie for helpful discussion, and Takako Matsui and Akiko Yamabe for technical assistance.

\section{References}

Brade, H. \& Galanos, C. (1983). Common lipopolysaccharide specificity: new type of antigen residing in the inner core region of $S-$ and R-form lipopolysaccharides from different families of Gramnegative bacteria. Infection and Immunity 42, 250-256.

BRADE, L. \& BRADE, H. (1985). A 28000-dalton protein of normal mouse serum binds specifically to the inner core region of bacterial lipopolysaccharide. Infection, and Immunity 50, 687-694.

Drewry, D. T., Symes, K. C., Gray, G. W. \& Wilkinson, S. G. (1975). Studies of polysaccharide fractions from the lipopolysaccharide of Pseudomonas aeruginosa NCTC 1999. Biochemical Journal 149, 93-106.

Evans, M. E., Pollack, M., Hardegen, N. J., Koles, N. L., Guelde, G. \& CHIA, J. K. S. (1990). Fluorescence-activated cell sorter analysis of binding by lipopolysaccharide-specific monoclonal antibodies to gram-negative bacteria. Journal of Infectious Diseases 162, 148-155.
Fisher, M. W., Devlin, H. B. \& Gnabasik, F. J. (1969). New immunotype schema for Pseudomonas aeruginosa based on protective antigens. Journal of Bacteriology 98, 835-836.

Fomsgaard, A., Conrad, R. S., Galanos, C., Shand, G. H. \& Hoiby, N. (1988). Comparative immunochemistry of lipopolysaccharides from typable and polyagglutinable Pseudomonas aeruginosa strains isolated from patients with cystic fibrosis. Journal of Clinical Microbiology 26, 821-826.

Hancock, R. E. W., Mutharia, L. M., Chan, L., Darveau, R. P., SPEerT, D. P. \& PIER, G. B. (1983). Pseudomonas aeruginosa isolates from patients with cystic fibrosis: a class of serum-sensitive, nontypable strains deficient in lipopolysaccharide $\mathrm{O}$ side chains. Infection and Immunity 42, 170-177.

Homma, J. Y. (1976). A new antigenic schema and live-cell slideagglutination procedure for the infrasubspecific serologic classification of Pseudomonas aeruginosa. Japanese Journal of Experimental Medicine 46, 329-336.

Klebba, P. E., Benson, S. A., Bala, S., Abdullah, T., Reid, J., Singh, S. P. \& NiKaIDO, H. (1990). Determinants of OmpF porin antigenicity and structure. Journal of Biological Chemistry 265, 68006810.

LAEMMLI, U. K. (1970). Cleavage of structural proteins during the assembly of the head of bacteriophage T4. Nature, London 227, 680685.

Liu, P. V., Matsumoto, H., Kusama, H. \& Bergan, T. (1983). Survey of heat-stable, major somatic antigens of Pseudomonas aeruginosa. International Journal of Systematic Bacteriology 33, 256-264.

Luderitz, O., Freudenberg, M. A., Galanos, C., Lehmann, V., RiETSCHEL, E. T. \& ShaW, D. H. (1982). Lipopolysaccharides of gram-negative bacteria. Current Topics in Membranes and Transport 17, 79-151.

Nelson, D., Neill, W. \& Poxton, I. R. (1990) A comparison of immunoblotting, flow cytometry and ELISA to monitor the binding of anti-lipopolysaccharide monoclonal antibodies. Journal of Immunological Methods 133, 227-233.

Pollack, M., Raubitschex, A. A. \& Larrick, J. W. (1987). Human monoclonal antibodies that recognize conserved epitopes in the corelipid A region of lipopolysaccharides. Journal of Clinical Investigation 79, 1421-1430.

Rietschel, E. T., Brade, H., Brade, L., KaCa, W., Kawahara, K., Linder, B., Luderitz, O., Tomita, T., Schade, U., Seydel, U. \& ZAHRINGER, U. (1985). Newer aspects of the chemical structure and biological activity of bacterial endotoxins. Progress in Clinical and Biological Research 189, 31-50.

Rowe, P. S. N. \& MEadow, P. M. (1983). Structure of the core oligosaccharide from the lipopolysaccharide of Pseudomonas aeruginosa PAC1R and its defective mutants. European Journal of Biochemistry 132, 329-337.

Teng, N. N. H., Lam, K. S., Riera, F. C. \& Kaplan, H. S. (1983). Construction and testing of mouse-human heteromyelomas for human monoclonal antibody production. Proceedings of the National Academy of Sciences of the United States of America 50, 499-506.

Terashima, M., Uezumi, I., Tomio, T., Kato, M., Irie, K., OKuda, T., Yoxota, S. \& NoguCHI, H. (1991) Protective human monoclonal antibody directed to the outer core region of Pseudomonas aeruginosa lipopolysaccharide. Infection and Immunity 59, 1-6.

TsaI, C.-M. \& Frasch, C. E. (1982). A sensitive stain for detecting lipopolysaccharides in polyacrylamide gels. Analytical Biochemistry 119, 115-119.

UCHIDA, K. \& Mizushima, S. (1987). A simple method for isolation of lipopolysaccharides from Pseudomonas aeruginosa and some other bacterial strains. Agricultural and Biological Chemistry 51, 3107-3114.

WiLkinson, S. G. (1983). Composition and structure of lipopolysaccharides from Pseudomonas aeruginosa. Reviews of Infectious Diseases 5, S941-S949.

Yokota, S., Ochi, H., OHTsuka, H., Kato, M. \& Noguchi, H. (1989). Heterogeneity of the L-rhamnose residues in the outer core of Pseudomonas aeruginosa lipopolysaccharide, characterized by human monoclonal antibodies. Infection and Immunity 57, 1691-1696.

YoKota, S., KaYA, S. \& NoGUCHI, H. (1990). Antigenic epitope in Pseudomonas aeruginosa lipopolysaccharide immunologically cross reactive with Escherichia coli $\mathrm{O} 26$ lipopolysaccharide. FEMS Microbiology Letters 68, 245-248. 